\title{
Oblique Incidence Ultrasonic Reflectometry Device Based on c-axis Tilted ScAIN Films for Evaluating Viscoelastic Properties of Liquids Above $100 \mathrm{MHz}$
}

\author{
Sarina Kinoshita \\ Waseda University \\ Takahiko Yanagitani ( $\nabla$ yanagitani@waseda.jp ) \\ Waseda University
}

\section{Research Article}

Keywords: ultrasonic reflectometry device, c-axis, ScAIN films, viscoelastic properties, liquids above 100 $\mathrm{MHz}$

Posted Date: January 18th, 2021

DOI: https://doi.org/10.21203/rs.3.rs-147045/v1

License: (c) (i) This work is licensed under a Creative Commons Attribution 4.0 International License.

Read Full License 


\title{
Oblique incidence ultrasonic reflectometry device based on c-axis tilted ScAIN films for evaluating viscoelastic properties of liquids above $100 \mathrm{MHz}$
}

\author{
Sarina Kinoshita ${ }^{1,2}$, and Takahiko Yanagitani $i^{1,2,3, *}$ \\ ${ }^{1}$ Waseda University, Graduate School of Advanced Science and Engineering, Tokyo, 169-8555, Japan \\ ${ }^{2}$ Kagami Memorial Research Institute for Material Science and Technology, Tokyo, 169-0051, Japan \\ ${ }^{3}$ JST CREST, Saitama, 332-0012, Japan \\ *yanagitani@waseda.jp
}

\section{ABSTRACT}

Ultrasound-based evaluation of fluid properties allows for real-time measurement of small amounts of liquid samples. The ultrasonic reflection method is used to obtain the complex reflection coefficient, which can be used to evaluate the viscoelastic properties of liquids. However, this method has not been used with shear waves at frequencies above $100 \mathrm{MHz}$ because shear-mode piezoelectric films are difficult to obtain at such frequencies. We propose using the oblique incidence reflectometry with quasi-shear waves excited by c-axis tilted scandium aluminum nitride (ScAlN) thin films to realize high-sensitivity evaluation of the viscoelastic properties of liquids in the above $100 \mathrm{MHz}$. In experiments, the shear elasticity and shear viscosity of glycerin solutions were estimated from their complex reflection coefficients.

\section{Introduction}

Ultrasound-based evaluation of fluid properties allows for the real-time measurement of small liquid samples.

This technique has attracted attention in the medical field for applications such as blood tests, where the fluid viscoelasticity may be used to detect diseases. The ultrasonic reflection method [1], [2] is used to obtain the complex reflection coefficient of a viscoelastic liquid, which can be used to evaluate viscoelastic properties such as the 
complex acoustic impedance, shear modulus, and shear viscosity. Although many studies have used this method [3]-

[6], shear waves at frequencies above $100 \mathrm{MHz}$ have not been considered because shear-mode piezoelectric films are difficult to obtain in this frequency range. In this study, we used the oblique incidence reflectometry with quasi-shear mode c-axis tilted scandium aluminum nitride (ScAlN) thin films to realize the high-sensitivity evaluation of the viscoelastic properties of liquids at frequencies above $100 \mathrm{MHz}$. Experiments were performed with glycerin solutions to demonstrate the performance of the proposed method.

\section{2. c-Axis tilted ScAIN}

Static piezoelectric constant $d_{33}$ in AlN films is increased by Sc doping [7]. We have previously reported, for the first-time, the experimental demonstration of electromechanical coupling coefficient of thickness-extensional mode $k_{\mathrm{t}}^{2}\left(k_{\mathrm{t}}^{2}=15 \%\right)$ in the c-axis oriented ScAlN film bulk acoustic wave resonator (FBAR), and that of thickness quasishear mode $k_{35}^{\prime}{ }^{2}\left(k_{35}^{\prime}{ }^{2}=10 \%\right)$ in the c-axis tilted ScAlN FBAR [8]. The theoretical electromechanical coupling coefficient of quasi thickness shear mode $\left(k_{35}^{\prime}{ }^{2}\right)$ and that of quasi thickness extensional mode $\left(k_{33^{\prime}}^{2}\right)$ in $\operatorname{ScAlN}$ and AlN were calculated using Caro's constants [9] as a function of c-axis tilt angle. The maximum $k_{35}^{\prime}{ }^{2}$ for $\mathrm{Sc}_{0.45} \mathrm{Al}_{0.55} \mathrm{~N}$ is much larger than that of AlN. $k_{35}^{\prime}$ reachs maximum at c-axis tilt angle of $35^{\circ}$.

\section{Measurement principle}

The principle of the proposed method can be described as follows. The complex reflection coefficient $\Gamma^{*}$ at the interface between a substrate and liquid is given by 
$\Gamma^{*}=\frac{Z_{\mathrm{S}}^{*} \cos \varphi-Z_{\mathrm{L}}^{*} \cos \psi}{Z_{\mathrm{S}}^{*} \cos \varphi+Z_{\mathrm{L}}{ }^{*} \cos \psi}$

where $Z_{\mathrm{S}}{ }^{*}, Z_{\mathrm{L}}{ }^{*}, \phi$, and $\psi$ are the complex acoustic impedances of the substrate and liquid and the angles of incidence and refraction, respectively, as shown in Fig. 1. If $\Gamma^{*}=\Gamma e^{-j \theta}$, then $Z_{\mathrm{L}}{ }^{*}$ is given by $Z_{\mathrm{L}}^{*}=\frac{\cos \varphi}{\cos \psi} \frac{1-\Gamma^{2}+j 2 \Gamma \sin \theta}{1+\Gamma^{2}+2 \Gamma \cos \theta} Z_{\mathrm{S}}^{*}$

If $\left|Z_{\mathrm{S}}{ }^{*}\right|>>\left|Z_{\mathrm{L}}{ }^{*}\right|$, then $\cos \psi=1$. In this study, because the incident angle of the trapezoidal silica glass prism was $60^{\circ}(\cos \phi=0.5)$, the measurement was twice as sensitive as that based on normal incidence $(\cos \phi=1)$. The theoretical $Z_{\mathrm{L}}{ }^{*}$ is given by $Z_{\mathrm{L}}^{*}=R_{\mathrm{L}}+j X_{\mathrm{L}}=\sqrt{\rho_{\mathrm{L}}\left(c_{44 \mathrm{~L}}+j \omega \eta_{\mathrm{L}}\right)}$,

where $\omega, \rho, c_{44 \mathrm{~L}}$, and $\eta$ are the angular frequency and the density, shear modulus, and viscosity of the fluid, respectively. $c_{44 \mathrm{~L}}$ and $\eta$ are given by

$$
c_{44 \mathrm{~L}}=\frac{R_{\mathrm{L}}^{2}-X_{\mathrm{L}}^{2}}{\rho_{\mathrm{L}}}
$$

and

$$
\eta_{\mathrm{L}}=\frac{2 R_{\mathrm{L}} X_{\mathrm{L}}}{\omega \rho_{\mathrm{L}}} .
$$

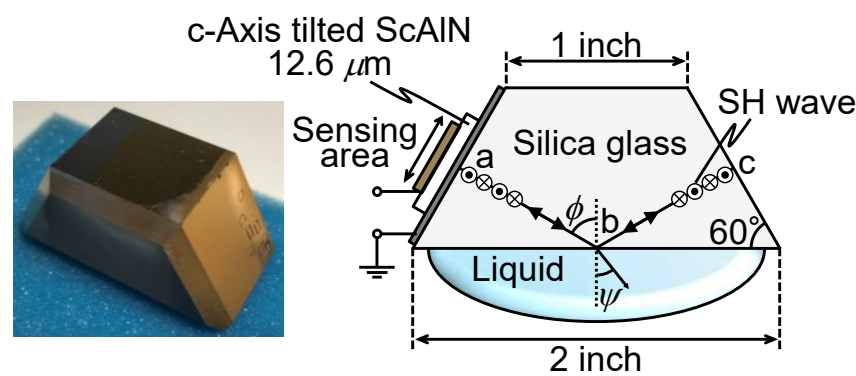

Fig. 1 Schematic of the trapezoidal silica glass prism. Also shown is the SH wave incidence at the interface between the substrate and fluid. 


\section{Methods}

\subsection{Fabrication of the trapezoidal silica glass prism}

A trapezoidal silica glass prism was used to realize oblique incidence of the SH wave. Figure 1 shows the schematic of the prism (top electrode: Au, piezoelectric film: c-axis tilted ScAlN, bottom electrode: Al, substrate: silica glass). The c-axis was tilted in the direction parallel to the interface to minimize mode conversion. The c-axis tilted ScAlN thin film was grown by glancing angle RF magnetron sputtering [10]. c-Axis tilt angle of $33^{\circ}$ was confirmed by XRD pole figure analysis.

\subsection{Analysis with a network analyzer}

The experimental system used in this study is shown in Fig. 1. The $n^{\text {th }}$ echo $S_{n \mathrm{~L}}$, which reflects at the interface between the substrate and liquid, was obtained from multiple-reflection data with a network analyzer (Agilent Technologies, E5071C). The $n^{\text {th }}$ echo $S_{n \mathrm{~A}}$ at the interface between the substrate and air was also obtained. The complex reflection coefficient $\Gamma^{*}$ is given by

$$
\Gamma^{*}=\left(\frac{S_{n \mathrm{~L}}{ }^{*}}{S_{n \mathrm{~A}}{ }^{*}}\right)^{\frac{1}{n}} .
$$

The complex acoustic impedance of the liquid $Z_{\mathrm{L}}{ }^{*}$ can be determined by using $\Gamma^{*}$ in Eq. (6) and the known $Z_{\mathrm{S}}{ }^{*}$. In this study, six glycerin solutions with different concentrations $(0,20,40,60,80$, and 97 wt.\%) were prepared as the liquid samples. The experiment was performed at $25^{\circ} \mathrm{C}$ in a thermostatic chamber. The temperature of the liquid was monitored with a platinum thermometer. 


\section{Results and discussion}

The properties of silica glass [11] and the glycerin solutions [12] were used to calculate $Z_{\mathrm{S}}{ }^{*}, c_{44}$ and $\eta$, where the substrate was assumed to be a purely elastic material $(\eta=0)$. Figure 2 shows the time domain response of the trapezoidal silica glass prism. The SH wave from the piezoelectric film was observed to propagate along the route abcba $(88 \mathrm{~mm})$ in Fig. 1. The peaks every $23.4 \mu$ s in Fig. 2 were those of the SH wave because the shear wave velocity of silica glass is $3768.9 \mathrm{~m} / \mathrm{s}$ [11]. Fig. 3 shows the frequency domain response of the insertion loss obtained by gating and Fourier transforming the first echo $\mathrm{SH}_{1}$. The efficiency of the $\mathrm{SH}$ wave excitation was highest at approximately $143 \mathrm{MHz}$.

The shear wave conversion loss [13] is measured to investigate $k_{35}^{\prime}{ }^{2}$ as shown in Fig. 4. The $k^{\prime} 35^{2}$ of c-axis tilted ScAlN film is determined to be $15.8 \%$ by comparing the experimental and theoretical conversion loss curves simulated by the electromechanical transmission line model (Mason's equivalent circuit model).

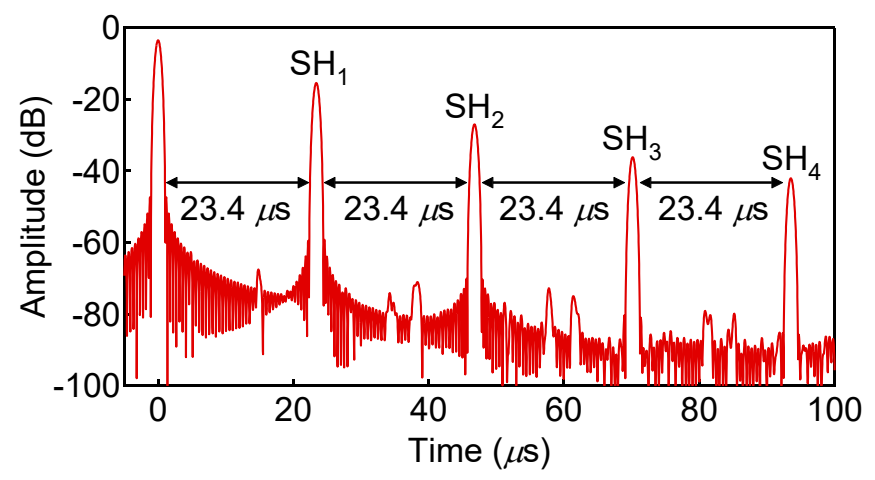

Fig. 2 Time response characteristics of the trapezoidal silica glass prism. 


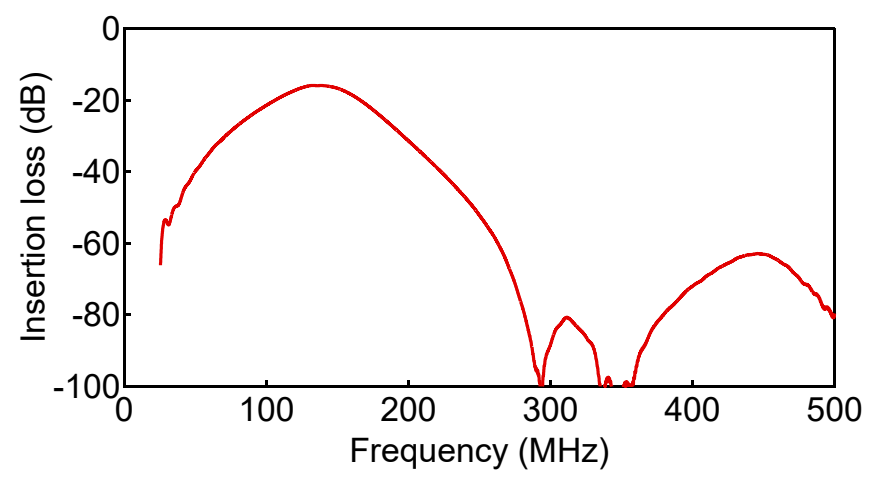

Fig. 3 Frequency characteristics for the insertion loss of the trapezoidal silica glass prism obtained by gating and Fourier transforming the first echo $\mathrm{SH}_{1}$.

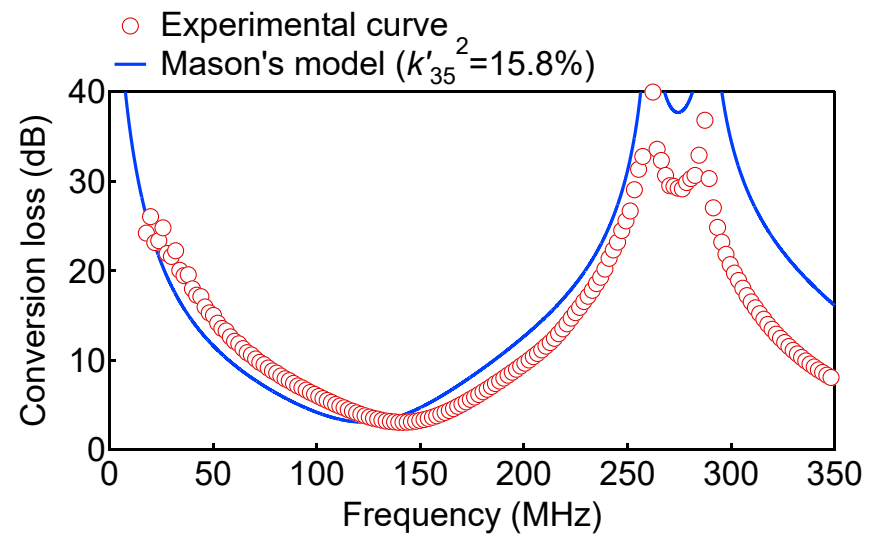

Fig. 4 Experimental shear wave conversion loss curves of c-axis tilted ScAlN films (red plot), which are in good agreement with the theoretical shear wave conversion loss curves (blue line) simulated by the electromechanical transmission line model (Mason's equivalent circuit model).

Figure 5 shows the measurement results for the shear elasticity $c_{44}$ and shear viscosity $\eta$ with the oblique incidence reflectometry. Figure 5 (a) shows that the amplitude of $\Gamma^{*}$ decreased monotonically with increasing glycerin concentration. However, Fig. 5 (b) shows a considerable variation in the phase of $\Gamma^{*}$, particularly for the 20 and 40 wt.\% glycerin solutions. This irregularity affected the calculations of the shear modulus and shear viscosity, as shown in Fig. 5 (c) and (d). The trapezoidal prism was so large that the propagation distance increased, and the temperature control of the entire prism was difficult, which caused errors in the result.

Next, we prepared smaller prism device to avoid these errors. The smaller prism is $6.4 \%$ volume of the previous 
one. As shown in Fig. 5, variation in the phase of $\Gamma^{*}$ were improved when smaller-type prism was used. This result in more precise measurement of the $c_{44}$ and $\eta$.
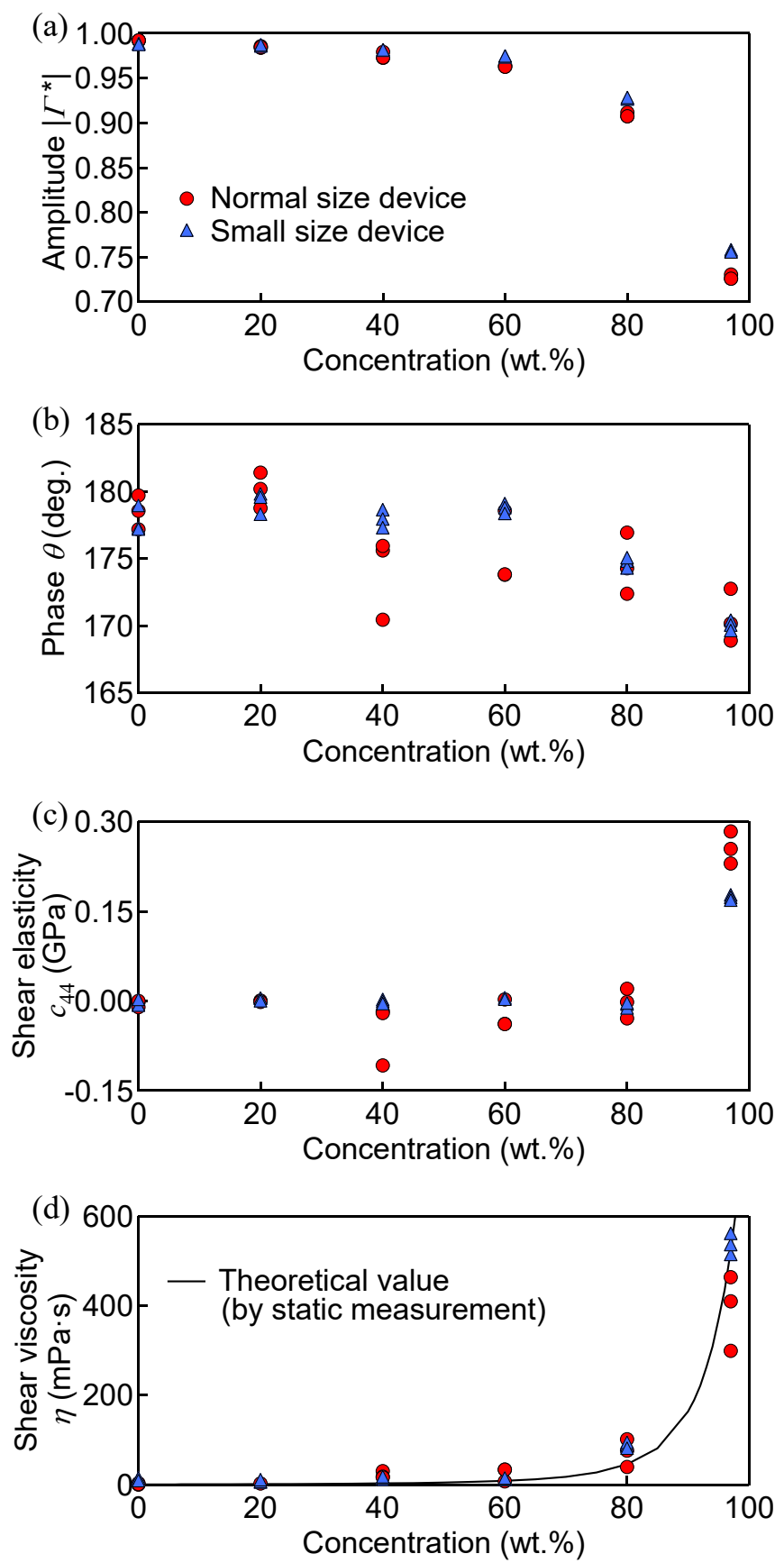

Fig. 5 Experimental results of the (a) amplitude and (b) phase of the complex reflection coefficient measured for the glycerin solutions. Calculated (c) shear elasticity and (d) shear viscosity of glycerin solutions based on the complex reflection coefficients. 


\section{Conclusion}

We used the complex reflection coefficient obtained with the oblique incidence reflectometry to measure the viscoelastic properties of a liquid. Measurements based on SH waves at frequencies above $100 \mathrm{MHz}$ were achieved with c-axis tilted ScAlN films.

\section{Acknowledgment}

The This work was supported by the JST CREST (No. JPMJCR20Q1) and KAKENHI (Grant-in-Aid for Scientific

Research No. 19H02202, and No. 18K19037).

\section{Data availability}

The datasets in the current study are available from the corresponding author on reasonable request.

\section{References}

[1] H. T. O’Neil, "Reflection and refraction of plane shear waves in viscoelastic media," Phys. Rev., vol. 75, pp. 928-935, 1949. DOI: 10.1103/PhysRev.75.928

[2] W. P. Mason, W. O. Baker, H. J. Mcskimin, and J. H. Heiss, "Measurement of shear elasticity and viscosity of liquids at ultrasonic frequencies,” Phys. Rev., vol. 75, pp. 936-946, 1949. DOI: 10.1103/PhysRev.75.936

[3] I. Alig, D. Lellinger, J. Sulimma, and S. Tadjbakhsch, "Ultrasonic shear wave reflection method for measurements of the viscoelastic properties of polymer films," Rev. Sci. Instrum., vol. 68, pp. 1536-1542, 1997. 
DOI: $10.1063 / 1.1147643$

[4] V. V. Shah and K. Balasubramaniam, "Measuring Newtonian viscosity from the phase of reflected ultrasonic shear wave," Ultrasonics, vol. 38, pp. 921-927, 2000. DOI: 10.1016/S0041-624X(00)00033-0

[5] M. S. Greenwood and J. A. Bamberger, "Measurement of viscosity and shear wave velocity of a liquid or slurry for on-line process control," Ultrasonics, vol. 39, pp. 623-630, 2002. DOI: 10.1016/S0041-624X(02)00372-4

[6] A. Yoneda and M. Ichihara, "Shear viscoelasticity of ultrasonic couplers by broadband reflectivity measurements," J. Appl. Phys., vol. 97, no. 054901, 2005. DOI: 10.1063/1.1850180

[7] M. Akiyama, T. Kamohara, K. Kano, A. Teshigahara, Y. Takeuchi, and N. Kawahara, "Enhancement of Piezoelectric Response in Scandium Aluminum Nitride Alloy Thin Films Prepared by Dual Reactive Cosputtering," Adv. Mater., vol. 21, pp. 593-596, 2009. DOI: 10.1002/adma.200802611

[8] T. Yanagitani, K. Arakawa, K. Kano, T. Akihiko, and M. Akiyama, in Proc. IEEE Ultrason. Symp., no. 5935791, pp. 2095-2098, 2010. DOI: 10.1109/ULTSYM.2010.5935791

[9] M. A Caro, S. Zhang, T. Riekkinen, M. Ylilammi, M. A Moram, O. Lopez-Acevedo, J. Molarius, and T. Laurila, "Piezoelectric coefficients and spontaneous polarization of ScAlN," J. Phys.: Condens. Matter, vol. 27, no. 245901, 2015. DOI: $10.1088 / 0953-8984 / 27 / 24 / 245901$

[10] T. Yanagitani, N. Morisato, S. Takayanagi, M. Matsukawa, and Y. Watanabe, "c-Axis zig-zag ZnO film ultrasonic transducers for designing longitudinal and shear wave resonant frequencies and modes," IEEE Trans. Ultrason., Ferroelectr., Freq. Contr., vol. 58, pp. 1062-1068, 2011. DOI: 10.1109/TUFFC.2011.1906

[11] J. Kushibiki, T. Wei, Y. Ohashi, and A. Tada, "Ultrasonic microspectroscopy characterization of silica glass," J. 
Appl. Phys., vol. 87, pp. 3113-3121, 2000. DOI: 10.1063/1.372307

[12] Physical Properties of Glycerin and Its Solutions, Glycerine Producers' Association, New York, (1969).

[13] T. Yanagitani, M. Suzuki, "Electromechanical coupling and gigahertz elastic properties of ScAlN films near phase boundary,” Appl. Phys. Lett., vol. 105, pp. 122907-1-122907-4, 2014. DOI: 10.1063/1.4896262

\section{Author Contributions}

S. Kinoshita performed the data analysis, T. Yanagitani supervised the work. S. Kinoshita and T. Yanagitani prepared the manuscript.

\section{Competing Interests}

The authors declare no competing interests.

\section{Additional information}

Correspondence and requests for materials should be addressed to T. Yanagitani and S. Kinoshita. 


\section{Figures}

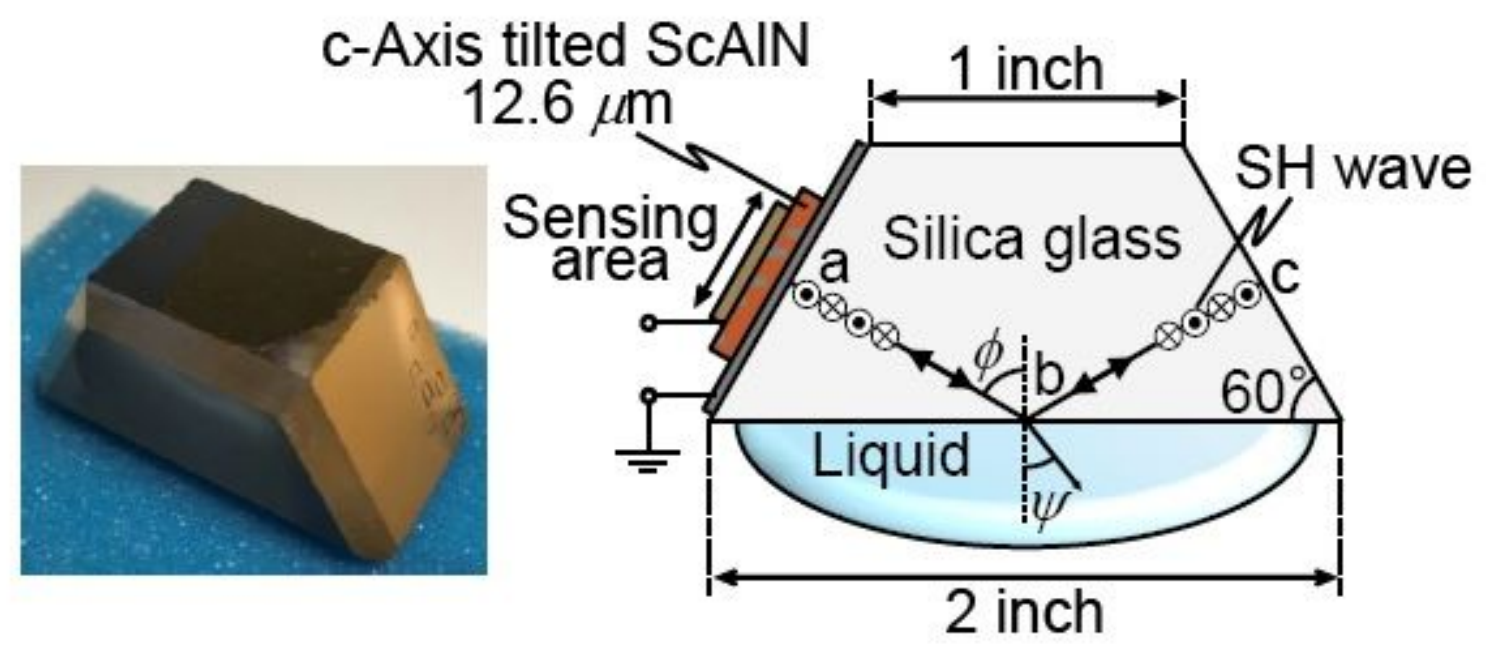

Figure 1

Schematic of the trapezoidal silica glass prism. Also shown is the $\mathrm{SH}$ wave incidence at the interface between the substrate and fluid.

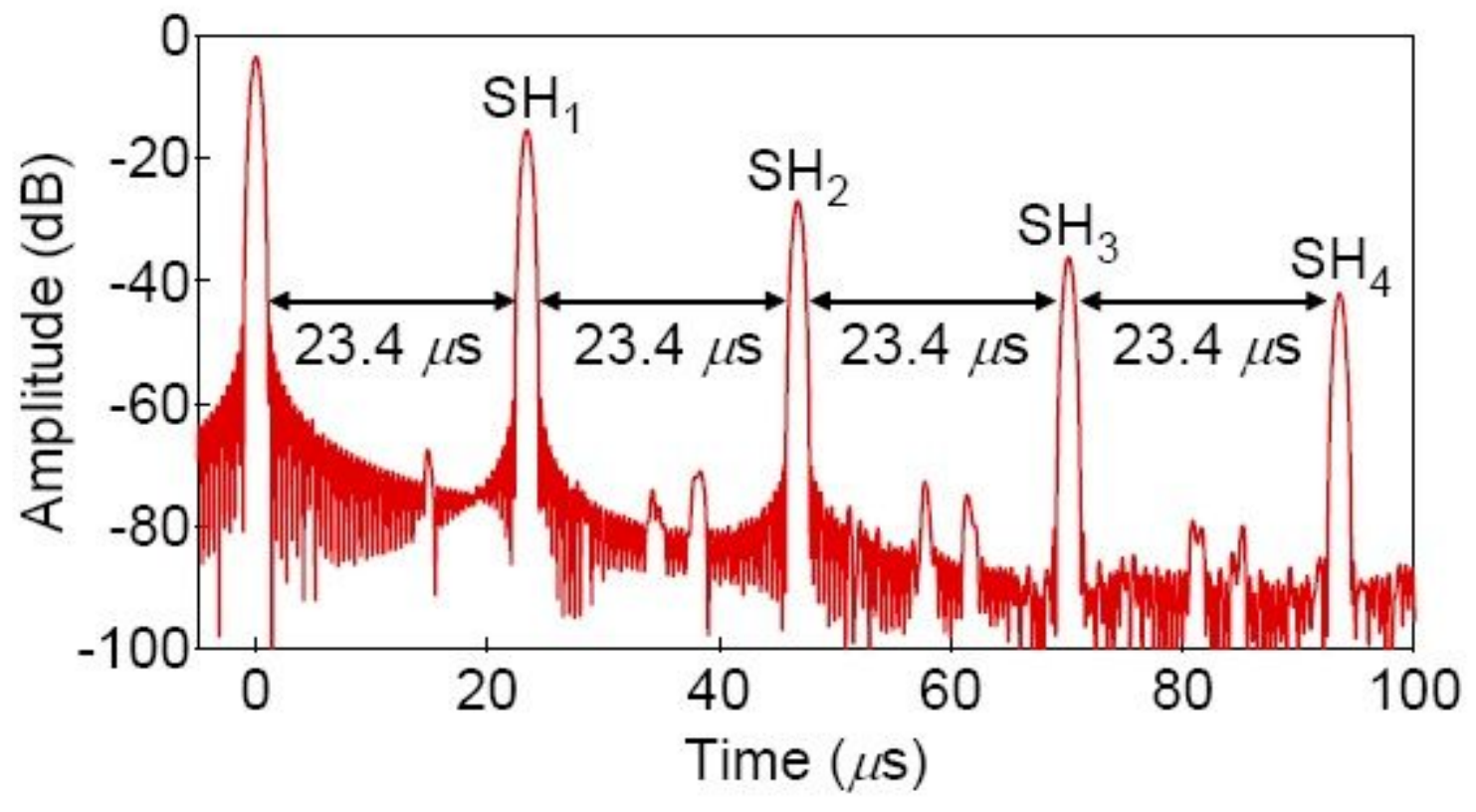

Figure 2

Time response characteristics of the trapezoidal silica glass prism. 


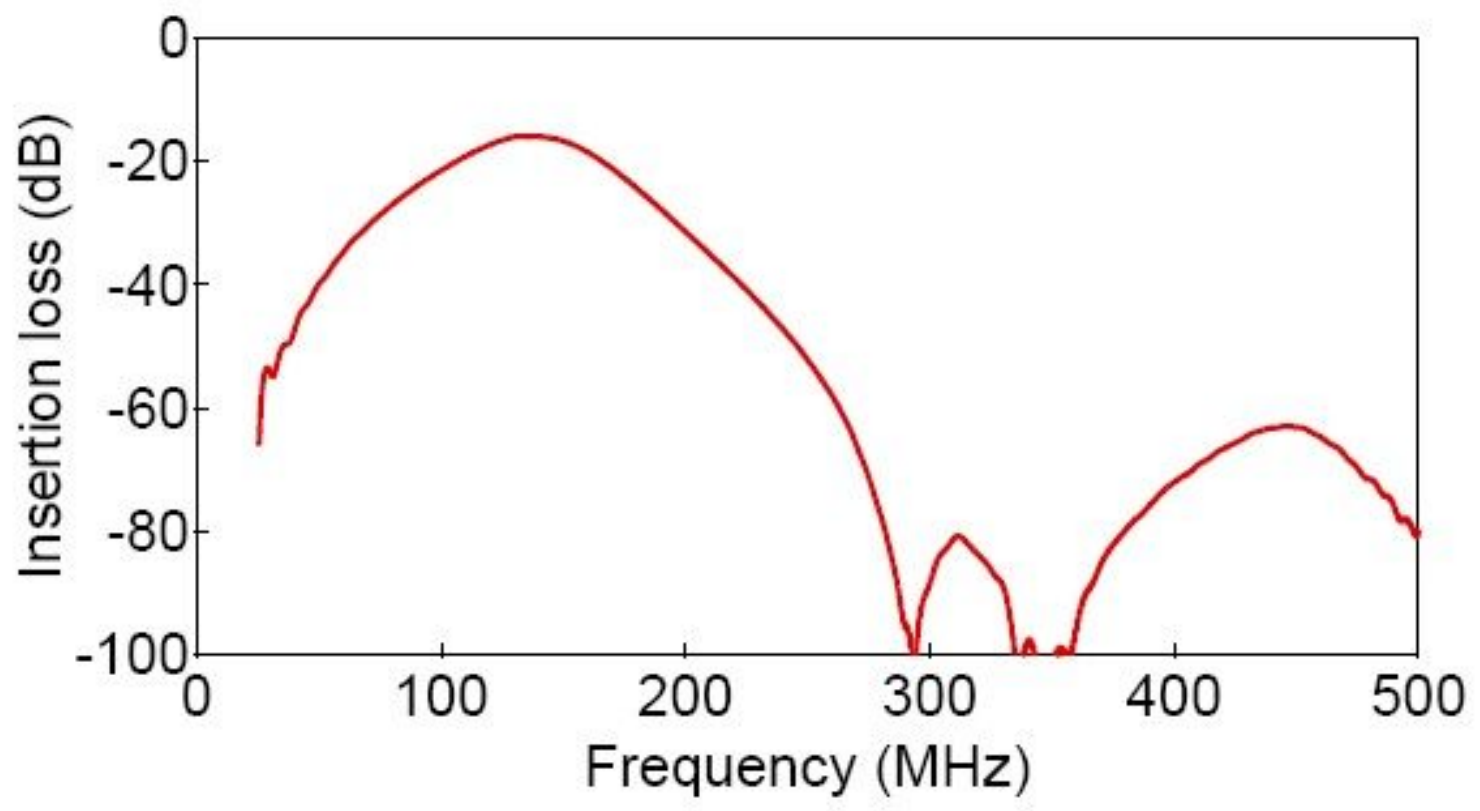

Figure 3

Frequency characteristics for the insertion loss of the trapezoidal silica glass prism obtained by gating and Fourier transforming the first echo SH1.

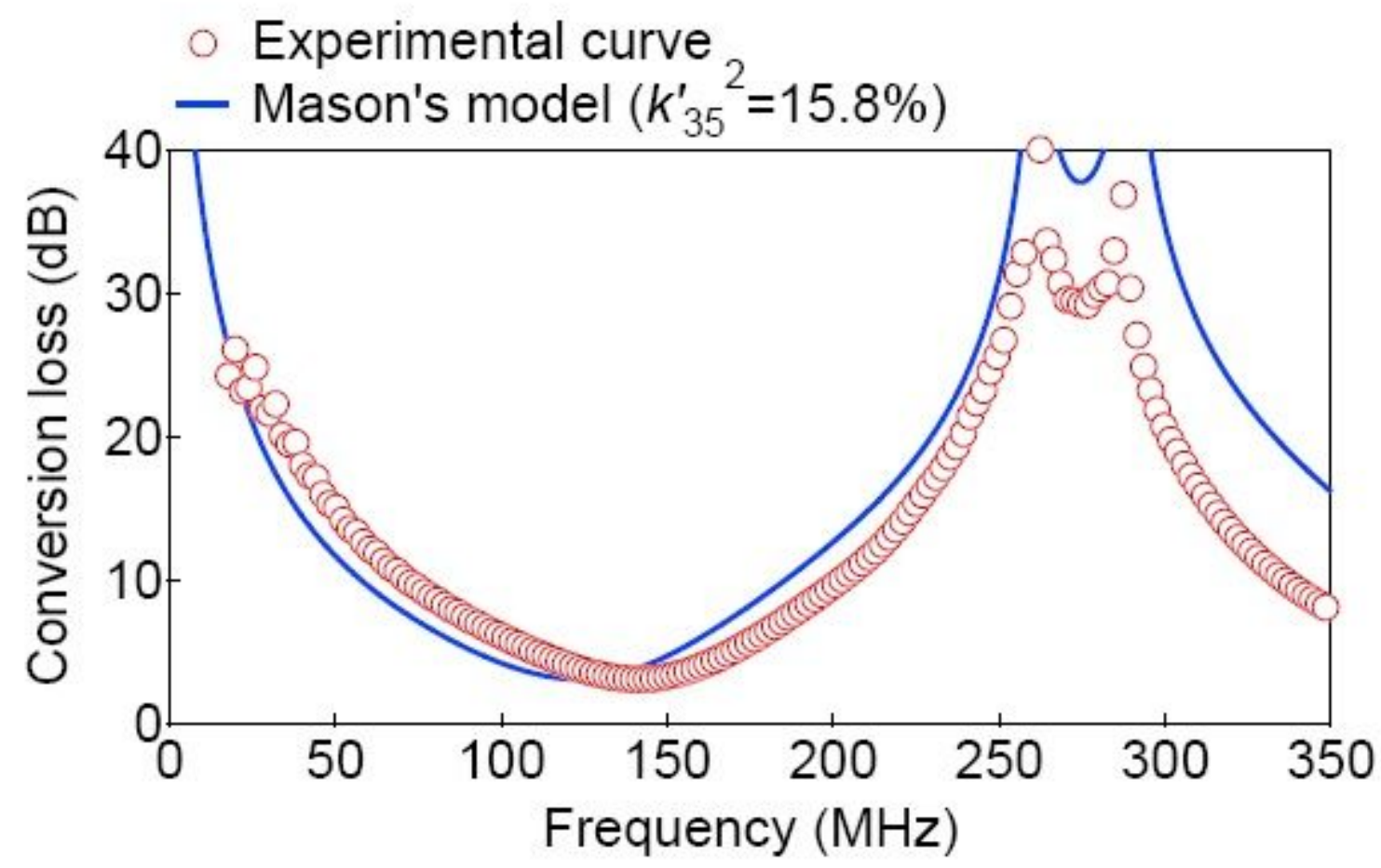

Figure 4 
Experimental shear wave conversion loss curves of c-axis tilted ScAIN films (red plot), which are in good agreement with the theoretical shear wave conversion loss curves (blue line) simulated by the electromechanical transmission line model (Mason's equivalent circuit model).
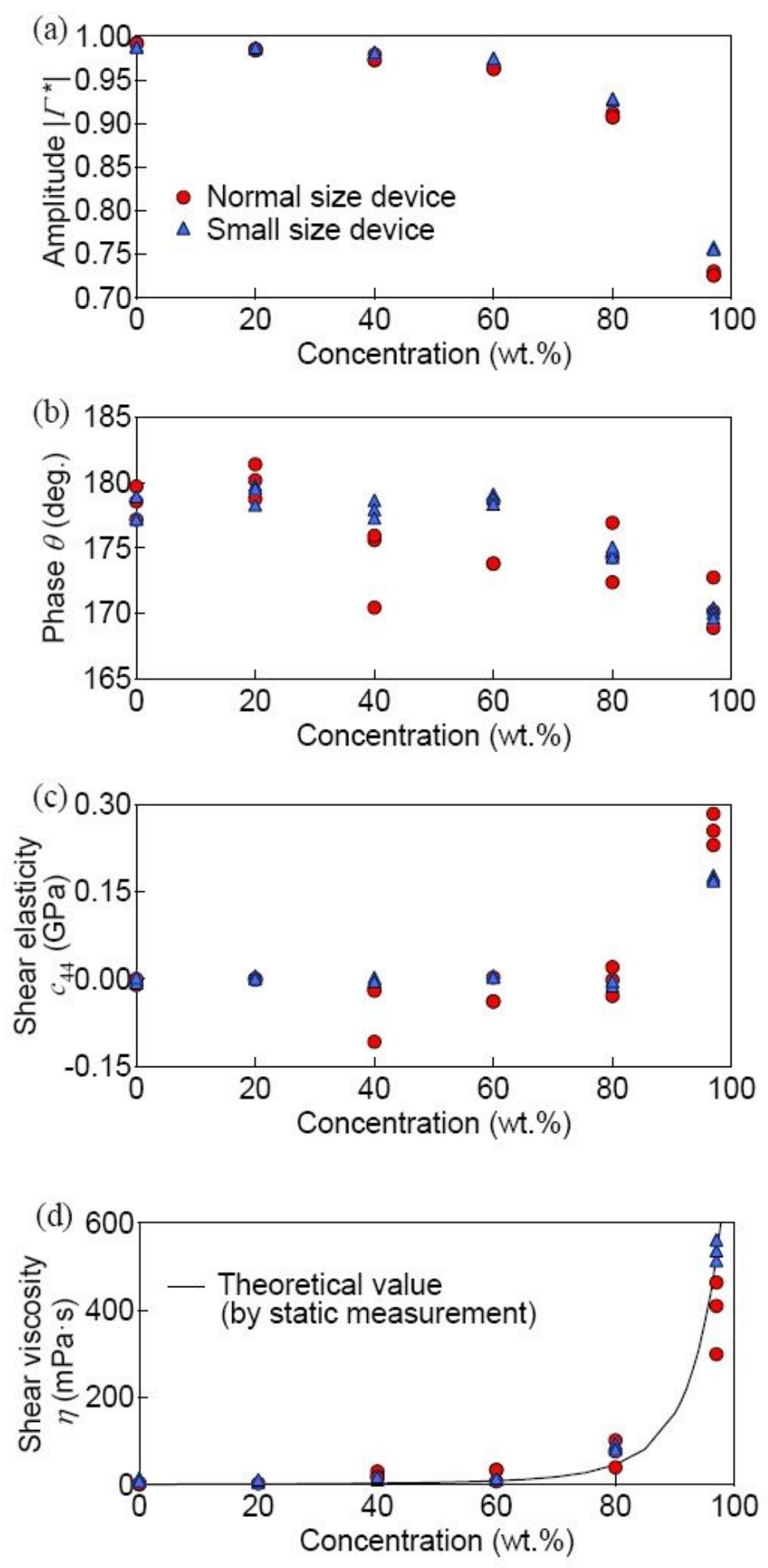

\section{Figure 5}

Experimental results of the (a) amplitude and (b) phase of the complex reflection coefficient measured for the glycerin solutions. Calculated (c) shear elasticity and (d) shear viscosity of glycerin solutions based 
on the complex reflection coefficients.

\section{Supplementary Files}

This is a list of supplementary files associated with this preprint. Click to download.

- 210113prismviscositySupplementaryMaterial.pdf 